\title{
Sistema de Gestión Ambiental en el Distrito de Ventanilla
}

\author{
Environmental management system in Ventanilla District
}

\author{
José Luis Alvarado Moreno' \\ Recibido: Junio 2019 - Aprobado: Julio 2019
}

\section{RESUMEN}

El Centro Poblado de Mi Perú en la actualidad cuenta con una población de 43,000 habitantes y posee una superficie total de $1.89 \mathrm{Km}^{2}$. Para esta localidad, una solución de gestión ambiental municipal, es presentada, la cual está orientada a objetivos, y trabaja bajo el enfoque del desarrollo sostenible. La gestión ambiental municipal es considerada como una importante herramienta de gestión para el municipio, por ello es necesario comprender que es realmente y que beneficios se logran, así como también, reconocer la importancia de incorporar variables y factores ambientales en la gestión municipal. En esta investigación se priorizaron tres aspectos: 1) los diagnósticos Ambientales, para determinar el nivel de calidad ambiental en el Centro Poblado Mi Perú; 2) el marco conceptual y teórico de la Gestión Ambiental Municipal, que rodea la gestión ambiental municipal, sus diferentes instrumentos, el área de aplicación del estudio; 3) el Sistema de Gestión Ambiental propuesto, del cual se presenta su arquitectura, funcionamiento y características. Finalmente se presentan los resultados, conclusiones y recomendaciones obtenidas producto del estudio realizado.

Palabras clave: Factores ambientales; índice de calidad ambiental; matriz de evaluación ambiental; sistema de gestión ambiental.

\begin{abstract}
The town Mi Peru currently has a population of 43,000 inhabitants and a total area of 1,89 $\mathrm{km}^{2}$. An alternative for a Municipal Environmental Management focus on the and with a clear focused of the community is presented. Municipal Environmental Management should be deemed as an important tool for the overall management of the municipality and to get we must internalize the concept and accept the inclusion of variables and environmental factors. In this research three aspects were prioritized: 1) the Environmental Diagnosis to define the environmental quality index in the town Mi Peru; 2) the conceptual and theoretical framework of the Municipal Environmental Management, the different instruments, the implementation of the study area; 3) The proposed Environmental Management System, which is defined as integrating its structure and operation. Finally, findings, conclusions and recommendations are described.
\end{abstract}

Keywords: Environmental Management System; environmental quality index; environmental assessment matrix; environmental factors.

\footnotetext{
1 Egresado de la Maestría en Gestión Integrada en Seguridad, Salud ocupacional y medio Ambiente, Facultad de Ingeniería Geológica, Minera, Metalúrgica y Geográfica, Universidad Nacional Mayor de San Marcos - Lima, Perú. E-mail: alvaradoambiental@hotmail.com 


\section{INTRODUCCIÓN}

Los Municipios son uno de los factores clave para lograr una gestión ambiental eficiente (Toledo, 2017), ya que es a nivel local donde se evidencian los principales problemas ambientales que padecen las pequeñas comunidades, del distrito de Ventanilla. Una de estas comunidades es el Centro Poblado Nuestra Señora de las Mercedes Mi Perú, ubicado en el distrito de Ventanilla, provincia constitucional del Callao (Municipalidad de Mi Perú, 2016).

Según los datos meteorológicos obtenidos de(Senamhi, 2018), el distrito de Ventanilla está clasificado como zona desértica semi cálida, con deficiencia de lluvias en todas las estaciones del año; presenta temperaturas no mayores a los $30^{\circ}$ centígrados en el verano, y con humedad en la temporada de invierno. En cuanto a la distribución urbana del territorio de Mi Perú, su zona central, está dividido por sectores, mientras que los asentamientos humanos se ubican en la zona periférica. Para (López Vásquez, 2012), la poca gestión ambiental por parte del municipio, acrecienta el problema, lo que trae consigo el deterioro del medio ambiente y por consiguiente condiciones de vida pobres en la zona.

Otro causante del problema, es la falta de comunicación entre las áreas encargadas de tratar los temas ambientales de la comunidad del propio municipio, ya que en la mayoría de casos estas trabajan de manera independiente y no en coordinación con las demás áreas, en lo que respecta a variables ambientales.

En atención a lo anteriormente expuesto se plantea como objetivo desarrollar un modelo conceptual y teórico ambiental para una propuesta de la Gestión Ambiental en el Centro Poblado de Mi Perú.

\section{MARCO TEÓRICO}

En el Perú, la conservación y protección del medio ambiente es una forma en la cual los municipios compiten entre sí, por tal motivo, un marco general se aprobó como medio para regular la gestión ambiental en la Municipalidad Provincial de San Martin (Alegre \& Chirinos, 2001), orientado hacia el desarrollo sostenible y la mejora continua de los factores críticos ambientales del distrito, con el objetivo de articular las políticas ambientales de las instituciones estatales que esten asociados a la gestión municipal, y con la ayuda de la participación ciudadana (Alegre C. M., 2002). Para esta misma ciudad (Ágreda, 2000) describe el Plan de Gestión Ambiental elaborado por el Consejo Ejecutivo Provincial para el Medio Ambiente de San Martín (CEPMA), su participación y la concertación local para una adecuada gestión ambiental.

En la Municipalidad de San Isidro viendo la necesidad de un marco legal claro y específico en materia de gestión ambiental que la regule, estableciendo lineamientos de política e instrumentos de gestión; se aprueban los lineamientos de gestión ambiental local en el distrito de San Isidro, considerando los siguientes factores: administración, control, mantenimiento y desarrollo del espacio geográfico y sus vecinos; concientizándolos sobre su responsabilidad en la conservación del ambiente. (Muncipalidad de San Isidro, 2008).
Desde los años 70 , se conoce la gestión ambiental como una reorientación del eco desarrollo y desarrollo sostenible, y como una herramienta de evaluación para solucionar los principales problemas ambientales, que día a día se van incrementando. Movimientos ambientalistas toman un rol protagónico, lo que provoca que el estado tome fuertes medidas para afrontar los problemas mencionados. (Muriel, 2006).

Para (Gonzales, 2001), considera la gestión ambiental como un proceso político-financiero, el cual se ocupa de organizar los diversos recursos, con el objetivo de proteger y preservar los recursos naturales, así como el medio ambiente.

Visto desde un enfoque de negocios, la normatividad de estandarización universal ISO, "Norma ISO 14001" de 1996, define la Gestión Ambiental de una organización (SGMA, según siglas utilizadas en español) como "la parte del sistema general de gestión que incluye la estructura organizativa, la planificación de las actividades, las responsabilidades, las prácticas, los procedimientos, los procesos y los recursos para desarrollar, implantar, llevar a efecto, revisar y mantener al día la política ambiental" (Sarde, 1999).

(Latorre, 2000) escribe que "La Gestión Ambiental puede considerarse como una tarea que comprende la evaluación, planificación, puesta en marcha, ejecución y evaluación del conjunto de acciones físicas, financieras, reglamentarias, institucionales, de participación, concertación, investigación y educación, con el fin de mejorar la calidad ambiental objeto de acción (entorno territorial de la empresa, proyecto de infraestructura, territorio de su jurisdicción)".

Ahora, (Serrano, 1997) menciona que "Por Gestión Ambiental entenderemos... toda intervención que busque la adecuación en las relaciones entre sociedad y su entorno natural, con independencia de los programas concretos que utilice para ello y, por lo tanto, con independencia de que los instrumentos gestores pertenezcan a la economía, a la política, a la ciencia, al derecho o a la administración ambiental".

En resumen, definimos gestión como la intervención planificada de las personas involucradas en la preservación del ambiente (Estado, empresarios, la sociedad), para lograr la resolución de un conflicto ambiental y para lo cual es necesario planificar e implementar una serie de actividades, así como definir índices para medir la gestión ambiental, como los índices de calidad de vida, donde (Cabrera, y otros, 2012) a partir de la calidad ambiental, se constituyen en un instrumento de gestión para la planificación y el control ambiental.

La Ley General del Ambiente (Ministerio del Ambiente, 2005) es la norma ordenadora del marco normativo legal para la gestión ambiental en el Perú. En ella se definen las reglas básicas para mantener un ambiente saludable equilibrado para el óptimo desarrollo de la vida. Además, dicha ley regula el desempeño de los deberes relacionados a la gestión ambiental, que incluye la mejora de la calidad de vida de la comunidad, mejora del 
ambiente urbano y rural, y preservación de la naturaleza. Los indicadores de gestión ambiental, son definidos como criterios para evaluar el comportamiento de una variable medioambiental. Detrás de cada indicador, siempre se tiene en cuenta la justificación del porque este es incluido y como será medido.

Según (Carmona \& Monsalve, 1999) un SIG es un sistema complejo de mapas, bases de datos e información básica y temática, que permite administrar los datos recogidos, realizando análisis de la información, según los requerimientos del escenario estudiado. Dentro de las funciones básicas podemos describir la captura de la información, esta se logra mediante procesos de digitalización, procesamiento de imágenes de satélites, fotografías, videos, entre otros. Estos sistemas complejos, son la herramienta informática más adecuada y extendida para la investigación y el trabajo profesional en Ciencias de la Tierra y Ambientales (Bosque, 1992).

\section{METODOLOGÍADE INVESTIGACIÓN}

El presente trabajo será una investigación no experimental descriptiva explicativa.

El Procedimiento que se seguirá para la realización de la investigación será según la figura 1 .

\subsection{HIPÓTESIS GENERAL}

El desarrollo de un modelo conceptual y teórico ambiental podrá permitir proponer una Gestión Ambiental en el Centro Poblado Mi Perú.

Variables Dependientes: Sistema de Gestión Ambiente Municipal

Variables Independientes: Instrumentos de Gestión Ambiental, Diagnósticos Ambiental, Diagnósticos Administrativos.

\subsection{UBICACIÓN Y AMBITO DE ESTUDIO}

El Centro Poblado Mi Perú se encuentra en el distrito de Ventanilla, provincia Constitucional del Callao, departamento de Lima. La Unidad de Análisis se ha tomado en cuenta toda el área que ocupa el Centro poblado de mi Perú.

\subsection{MUESTRA}

Para efectos del presente estudio, se consideró el Centro Poblado Mi Perú, está distribuido en 13 sectores (A, B, C, D, E, F, G, H, I, J, K, M, N) y 15 asentamientos humanos.

Para la selección de la muestra se consideran las áreas verdes de los sectores y asentamientos humanos, así mismo las industrias y talleres comprendió dentro del centro poblado Mi Perú

\subsection{METODOLOGÍA DE REVISIÓN Y RECOPILACIÓN DE INFORMACIÓN}

La investigación se inició con una fase preliminar de recopilación o de documentación de la información que se obtiene referente a la temática en estudio, lo que permitió formular las hipótesis correspondientes.

Referente a las fuentes bibliográficas, se inició con una búsqueda no especializada, continuándose con la búsqueda de investigaciones especializadas, investigaciones institucionales, asesoría de diferentes profesionales de diversas instituciones, trabajos de investigación (Tesis, trabajos especiales, entre otros), revistas científicas, leyes, finalmente se realizó una búsqueda de publicaciones, documentos y artículos en línea relacionados al desarrollo rural sostenible mediante la recuperación de andenes.

En los entes públicos se recopiló y evaluó los estudios existentes, cartográfica e imágenes satélites, mapas, carta nacional ubicándose los diferentes sectores y asentamientos humanos, objetivo del estudio. A continuación, se realizó el proceso de revisión de la recopilación del material bibliográfico, clasificándolo y ordenándolo de acuerdo a la información que se relaciona con el área de estudio.

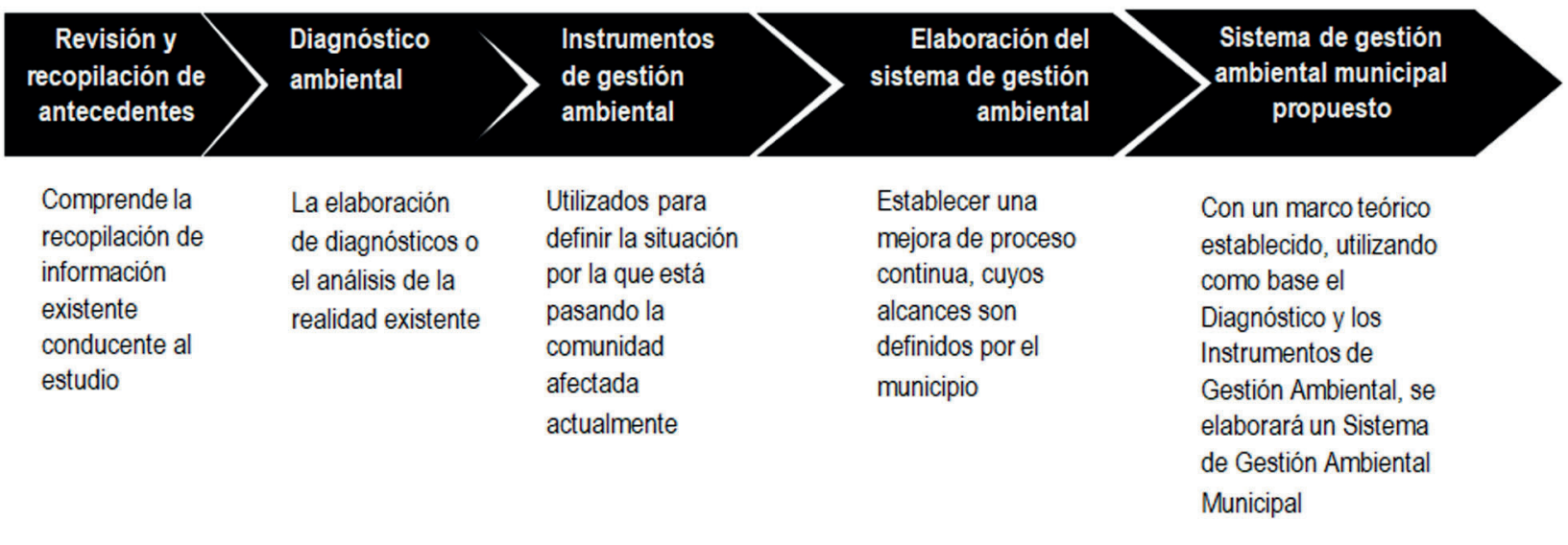

Figura 1. Metodología de Investigación 


\subsection{METODOLOGÍA DEL TRABAJO DE CAMPO}

Posterior a la revisión y recopilación de la información, se programó las respectivas salidas de campo para determinar el esquema de estudio, durante esta fase con la colaboración de algunos miembros de la municipalidad se identificó y cuantificó el estado actual de los sectores y asentamientos humanos, lo cual se utilizó instrumentos como el GPS (Global Positioning System: sistema de posicionamiento global), brújula tipo Brunton, huincha o cinta métrica, cámara fotográfica, libreta de campo, lápices de color, recipientes para tomar muestras; además de la información proporcionada por las imágenes satelitales, la cartográfica adquirida del Instituto Geográfico Nacional y del procesamiento de la información en el entorno del sistema de información geográfica (SIG).

\subsection{ANALISIS AMBIENTAL}

Se define la calidad Ambiental de cada una de los sectores y asentamiento humanos dentro de la zona de estudio. Para ello se determinó un Índice de Calidad Ambiental, el que está en función de los factores ambientales seleccionado para este propósito.

Factores Ambientales seleccionados

- Densidad de Áreas Verdes

- Densidad de Habitantes

- Densidad de Viviendas

- Influencia de Industrias y Talleres

Se seleccionaron estos factores por su aporte, positivo o negativo en la calidad de vida y ambiental de la comunidad.

Factores Ambientales no seleccionados

- Calidad Hidrológica

- Calidad Atmosférica

- Calidad de Suelos

La razón por la que no se escogieron estas variables ambientales se debe a la poca información específica que se tiene en la actualidad. Por ejemplo, no existe un control en la medición de los contaminantes que componen la contaminación atmosférica. Sin duda esto se presenta como un gran desafío hacia el futuro para poder contar con esta información. Para definir el índice de calidad ambiental, primero es la recolección de los datos del centro poblado, luego un ajuste estadístico es necesario para que no se presenten descompensaciones de algunos factores, como último paso, y haciendo uso del sistema de información geográfico, para representar los datos y dividir el área estudiada en zonas homogéneas según la calidad ambiental.

\subsection{DETERMINACIÓN DEL ÍNDICE DE CALIDAD AMBIENTAL}

La metodología utilizada por (Spiegel \& Yassi, 1997), para la designación del Índice de Calidad Ambiental contiene los siguientes pasos y relaciones estadísticas:

a. Estandarizar los resultados de los diferentes factores mediante una distribución normal utilizando la siguiente fórmula:

$$
\mathrm{z}=\frac{\mathrm{X}-\overline{\mathrm{X}}}{\alpha}
$$

Donde: $\mathrm{X}=$ Variable

$$
\begin{aligned}
& \bar{X}=\text { Promedio } \\
& \alpha=\text { Desviación estándar }
\end{aligned}
$$

b. Establecer el número de clases o rangos por medio de la ecuación de Brook Carruthers

$$
\text { N. }{ }^{\circ} \text { de Clases }=1+3.3^{*} \log (n)
$$

$\mathrm{n}=$ Número de unidades vecinales

N. ${ }^{\circ}$ de clases zona centro $=1+3,3+\log (27)$

N. ${ }^{\circ}$ de clases zona centro $=5,72 \approx 6$

c. Se describe la estructura de clases de los factores ambientales, donde se indica los valores máximo y mínimo y rango de clase (Ver tabla 1).

d. Se definen los factores ambientales caracterizándolas con una letra

A $=$ Densidad de Áreas Verdes

$\mathrm{B}=$ Densidad de Habitantes

\begin{tabular}{|c|c|c|c|c|c|c|c|c|}
\hline \multirow{2}{*}{ Rango } & \multicolumn{2}{|c|}{ Densidad de Áreas Verde } & \multicolumn{2}{|c|}{ Densidad de Habitantes } & \multicolumn{2}{|c|}{ Densidad de Vivienda } & \multicolumn{2}{|c|}{$\begin{array}{l}\text { Influencia de las Industrias y } \\
\text { Talleres }\end{array}$} \\
\hline & Mínimo & Máximo & Mínimo & Máximo & Mínimo & Máximo & Mínimo & Máximo \\
\hline 1 & 0.000 & 0.027 & 0.90 & 1.10 & -0.33 & 0.31 & -0.56 & 0.34 \\
\hline 2 & 0.027 & 0.054 & 1.1 & 1.30 & 0.31 & 0.95 & 0.34 & 1.24 \\
\hline 3 & 0.054 & 0.081 & 1.3 & 1.50 & 0.95 & 1.59 & 1.24 & 2.14 \\
\hline 4 & 0.081 & 0.108 & 1.5 & 1.70 & 1.59 & 2.23 & 2.14 & 3.04 \\
\hline 5 & 0.108 & 0.135 & 1.7 & 1.90 & 2.23 & 2.87 & 3.04 & 3.94 \\
\hline 6 & 0.135 & 0.163 & 1.9 & 2.11 & 2.87 & 3.52 & 3.94 & 4.84 \\
\hline
\end{tabular}

$\mathrm{C}=$ Densidad de Viviendas

$\mathrm{D}=$ Influencia de Industrias y Talleres

Tabla 1. Amplitud de intervalos de los Factores Ambientales 
Una vez definidos los factores se agrupan por rangos con su respectivo valor máximo y mínimo.

e. Se define la relación matemática que representará el Índice de Calidad Ambiental

Índice de Calidad Ambiental $(\mathrm{INCA})=\mathrm{A}-(\mathrm{B}+\mathrm{C}+\mathrm{D})$

f. Determinar el intervalo de variación del Índice de Calidad Ambiental

De acuerdo a los resultados de la relación matemática utilizada, el intervalo e índice de calidad ambiental que se configura es el siguiente:

$\begin{array}{llr}\text { Valor Máximo } & = & 4 \\ \text { Valor mínimo } & = & -12 \\ \text { Recorrido de la Variable } & = & 16 \\ \text { Número de Clases } & = & 6\end{array}$

Tabla 2. Intervalos de variación para medir la calidad ambiental

\begin{tabular}{|l|l|l|}
\hline \multicolumn{1}{|c|}{ Atributo } & \multicolumn{1}{|c|}{ Descripción } & \multicolumn{1}{c|}{ Intervalo } \\
\hline Muy Bueno & Muestra un Índice de Calidad Ambiental óptimo & De 1 a 4 \\
\hline Bueno & Muestra un Índice de Calidad más que regular & De -3 a 0 \\
\hline Regular & $\begin{array}{l}\text { Muestra un Índice de Calidad Ambiental discreto, } \\
\text { de precaución }\end{array}$ & De -7 a -4 \\
\hline Malo & $\begin{array}{l}\text { Muestra un Índice de Calidad Ambiental deficiente, } \\
\text { preocupante }\end{array}$ & De -12 a -8 \\
\hline
\end{tabular}

\section{RESULTADOS Y DISCUSIÓN}

Después de recoger y procesar los datos sobre las viviendas, habitantes, aéreas verdes e industrias y talleres; se consiguió un valor de rango, según los intervalos de la Tabla 1, para cada una de las 4 variables estudiadas (Ver tabla 3 ).

La tabla 4 muestra el índice de calidad ambiental calculado para cada unidad vecinal, así como el atributo correspondiente según la escala definida en la Tabla 2.

Tabla 3. Valores de Rango para las variables A, B, C y D

\begin{tabular}{|c|c|c|c|c|c|c|c|c|c|c|c|c|c|}
\hline $\mathbf{N}^{\circ}$ & Nombres & $\begin{array}{l}\text { Densidad } \\
\text { AV/Sup. }\end{array}$ & $\mathbf{z}$ & A & $\begin{array}{l}\text { Densidad } \\
\text { Viv/Sup. }\end{array}$ & $\mathbf{z}$ & B & $\begin{array}{l}\text { Densidad } \\
\text { Hab/Sup. }\end{array}$ & $\mathbf{z}$ & C & $\begin{array}{l}\text { Infl. } \\
\text { Ind. }\end{array}$ & $\mathbf{z}$ & D \\
\hline 1 & AA.HH. 7 de junio & 0.00 & 0.000 & 1 & 7447 & 0.53 & 3 & 32170.21 & 0.77 & 4 & 2 & -0.51 & 1 \\
\hline 2 & AA.HH. Las Terrazas & 0.00 & 0.000 & 1 & 6087 & 1.51 & 4 & 26086.96 & 0.18 & 4 & 10 & -0.40 & 1 \\
\hline 3 & Sector K & 0.00 & 0.000 & 1 & 5581 & -0.09 & 2 & 24651.16 & 0.04 & 3 & 2 & -0.56 & 1 \\
\hline 4 & Sector C & 0.03 & 0.020 & 1 & 5975 & 0.03 & 2 & 35448.04 & 1.09 & 5 & 12 & -0.23 & 1 \\
\hline 5 & Sector B & 0.01 & 0.000 & 1 & 5867 & 0.00 & 2 & 33566.27 & 0.90 & 5 & 30 & 0.31 & 1 \\
\hline 6 & Sector A & 0.02 & 0.001 & 1 & 6512 & 0.21 & 3 & 28956.11 & 0.46 & 4 & 2 & -0.56 & 1 \\
\hline 7 & Sector I & 0.00 & 0.000 & 1 & 6688 & 0.27 & 3 & 31250.00 & 0.68 & 4 & 26 & 0.21 & 1 \\
\hline 8 & AA.HH. Villa Emilia & 0.00 & 0.000 & 1 & 6108 & 0.08 & 2 & 27592.81 & 0.32 & 4 & 30 & -0.16 & 1 \\
\hline 9 & Sector J & 0.02 & 0.001 & 1 & 6076 & 0.07 & 2 & 30379.75 & 0.59 & 4 & 20 & 0.03 & 1 \\
\hline 10 & Sector G & 0.02 & 0.001 & 1 & 1064 & -1.61 & 1 & 5319.15 & -1.84 & 1 & 200 & 4.84 & 6 \\
\hline 11 & Sector D & 0.00 & 0.000 & 1 & 5966 & 0.03 & 2 & 23625.00 & -0.06 & 3 & 36 & 0.43 & 2 \\
\hline 12 & Sector $\mathrm{E}$ & 0.02 & 0.001 & 1 & 6158 & 0.10 & 2 & 24368.40 & 0.01 & 3 & 4 & -0.44 & 1 \\
\hline 13 & Sector M & 0.17 & 0.163 & 6 & 2294 & -1.20 & 1 & 11215.69 & -1.27 & 1 & 4 & -0.52 & 1 \\
\hline 14 & Sector F & 0.00 & 0.000 & 1 & 6890 & 0.34 & 2 & 33942.86 & 0.94 & 5 & 36 & 0.70 & 2 \\
\hline 15 & Sector N & 0.00 & 0.000 & 1 & 9931 & 1.36 & 4 & 45977.01 & 2.11 & 6 & 4 & -0.50 & 1 \\
\hline 16 & Sector $\mathrm{H}$ & 0.01 & 0.000 & 1 & 4987 & -0.29 & 2 & 18987.34 & -0.51 & 3 & 2 & -0.56 & 1 \\
\hline 17 & AA.HH. 13 de noviembre & 0.00 & 0.000 & 1 & 7889 & 0.68 & 3 & 33333.33 & 0.88 & 5 & 2 & -0.05 & 1 \\
\hline 18 & AA.HH. Señor de Luren & 0.00 & 0.000 & 1 & 16389 & 3.52 & 6 & 44444.44 & 1.96 & 6 & 4 & -0.05 & 1 \\
\hline 19 & AA.HH. Super Manzana & 0.00 & 0.000 & 1 & 5294 & -0.19 & 2 & 24705.88 & 0.04 & 3 & 2 & -0.32 & 1 \\
\hline 20 & AA.HH. Virgen del Rosario & 0.00 & 0.000 & 1 & 5000 & -0.29 & 2 & 15000.00 & -0.90 & 2 & 2 & -0.25 & 1 \\
\hline 21 & AA.HH. San Martin & 0.00 & 0.000 & 1 & 4833 & -0.35 & 2 & 23333.33 & -0.09 & 3 & 2 & 0.23 & 1 \\
\hline 22 & AA.HH. Santa Rosa & 0.00 & 0.000 & 1 & 4880 & -0.33 & 2 & 26000.00 & 0.17 & 4 & 12 & 0.61 & 2 \\
\hline 23 & AA.HH. El Arenal & 0.00 & 0.000 & 1 & 2250 & -1.21 & 1 & 9090.91 & -1.48 & 1 & 2 & -0.51 & 1 \\
\hline 24 & AA.HH. Hijos del Perú & 0.00 & 0.000 & 1 & 6000 & 0.04 & 2 & 18750.00 & -0.54 & 3 & 2 & -0.41 & 1 \\
\hline 25 & AA.HH. Villa del Mar & 0.00 & 0.000 & 1 & 2926 & -0.99 & 1 & 8088.24 & -1.57 & 1 & 6 & -0.40 & 1 \\
\hline 26 & AA.HH. Las Colinas & 0.00 & 0.000 & 1 & 9000 & 1.05 & 4 & 20000.00 & -0.41 & 3 & 2 & -0.11 & 1 \\
\hline 27 & AA.HH. Chavinillo & 0.00 & 0.000 & 1 & 2481 & -1.14 & 1 & 11503.74 & -1.24 & 1 & 14 & -0.35 & 1 \\
\hline 28 & AA.HH. Villa Escudero & 0.00 & 0.000 & 1 & 3837 & -0.68 & 2 & 11627.91 & -1.23 & 1 & 10 & -0.47 & 1 \\
\hline
\end{tabular}


Tabla 4. Resultado Índice de Calidad Ambiental

\begin{tabular}{|c|c|c|c|c|c|c|c|}
\hline $\mathrm{N}^{\circ}$ & Nombres & Rango A & Rango B & Rango C & Rango D & Calidad Ambiental & Atributo \\
\hline 1 & AA.HH. 7 de junio & 1 & 3 & 4 & 1 & -7 & Regular \\
\hline 2 & AA.HH. Las Terrazas & 1 & 4 & 4 & 1 & -8 & Malo \\
\hline 3 & Sector K & 1 & 2 & 3 & 1 & -5 & Regular \\
\hline 4 & Sector C & 1 & 2 & 5 & 1 & -7 & Regular \\
\hline 5 & Sector B & 1 & 2 & 5 & 1 & -7 & Regular \\
\hline 6 & Sector A & 1 & 3 & 4 & 1 & -7 & Regular \\
\hline 7 & Sector I & 1 & 3 & 4 & 1 & -7 & Regular \\
\hline 8 & AA.HH. Villa Emilia & 1 & 2 & 4 & 1 & -6 & Regular \\
\hline 9 & Sector J & 1 & 2 & 4 & 1 & -6 & Regular \\
\hline 10 & Sector G & 1 & 1 & 1 & 6 & -7 & Regular \\
\hline 11 & Sector D & 1 & 2 & 3 & 2 & -7 & Regular \\
\hline 12 & Sector E & 1 & 2 & 3 & 1 & -5 & Regular \\
\hline 13 & Sector M & 6 & 1 & 1 & 1 & 3 & Muy Bueno \\
\hline 14 & Sector $F$ & 1 & 2 & 5 & 2 & -8 & Malo \\
\hline 15 & Sector $\mathrm{N}$ & 1 & 4 & 6 & 1 & -10 & Malo \\
\hline 16 & Sector $\mathrm{H}$ & 1 & 2 & 3 & 1 & -5 & Regular \\
\hline 17 & AA.HH. 13 de noviembre & 1 & 3 & 5 & 1 & -8 & Malo \\
\hline 18 & AA.HH. Señor de Luren & 1 & 6 & 6 & 1 & -12 & Malo \\
\hline 19 & AA.HH. Super Manzana & 1 & 2 & 3 & 1 & -5 & Regular \\
\hline 20 & AA.HH. Virgen del Rosario & 1 & 2 & 2 & 1 & -4 & Regular \\
\hline 21 & AA.HH. San Martin & 1 & 2 & 3 & 1 & -5 & Regular \\
\hline 22 & AA.HH. Santa Rosa & 1 & 2 & 4 & 2 & -7 & Regular \\
\hline 23 & AA.HH. El Arenal & 1 & 1 & 1 & 1 & -2 & Bueno \\
\hline 24 & AA.HH. Hijos del Perú & 1 & 2 & 3 & 1 & -5 & Regular \\
\hline 25 & AA.HH. Villa del Mar & 1 & 1 & 1 & 1 & -2 & Bueno \\
\hline 26 & AA.HH. Las Colinas & 1 & 4 & 3 & 1 & -7 & Regular \\
\hline 27 & AA.HH. Chavinillo & 1 & 1 & 1 & 1 & -2 & Bueno \\
\hline 28 & AA.HH. Villa Escudero & 1 & 2 & 1 & 1 & -3 & Bueno \\
\hline
\end{tabular}

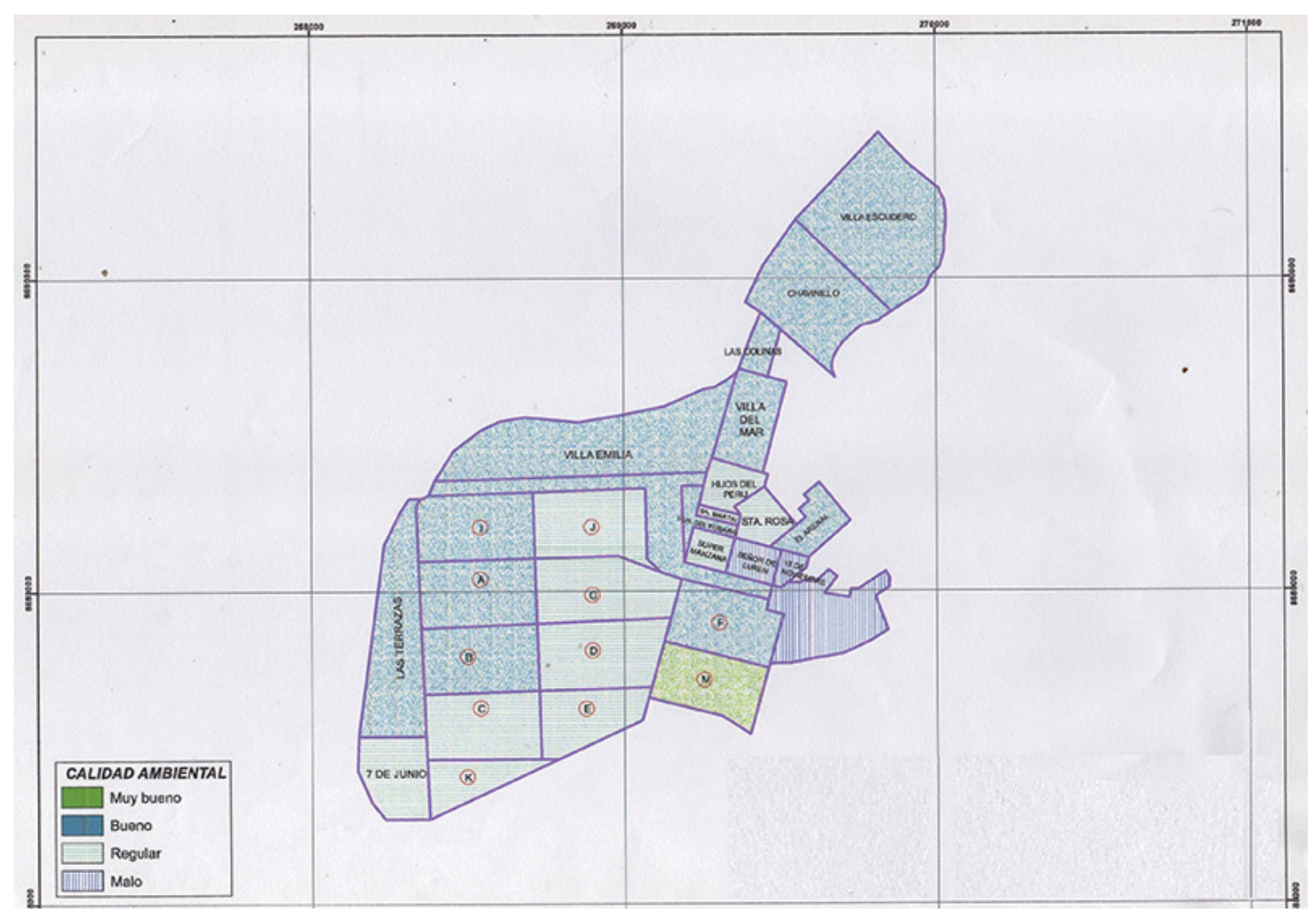

Figura 2. Calidad Ambiental del Centro Poblado Mi Perú 
Donde:

Calidad Ambiental Muy Buena: Esta zona se caracterizó por bajos índices de densidad de habitantes y viviendas, casi nula influencia industrial, por el contrario, presentó altos índices de áreas verdes en general.

Calidad Ambiental Buena: Esta zona se caracterizó por tener un índice medio de densidad de habitantes y vivienda, también una baja influencia de industrias y talleres, y presentó índices altos de áreas verdes en general.

Calidad Ambiental Regular: Esta zona presentó moderada influencia industrial, índices medios de densidad de habitantes y de vivienda, y baja presencia de áreas verdes en general.

Calidad Ambiental Mala: Esta zona se caracterizó por presentar las más adversas condiciones en cuanto a los factores ambientales estudiados.

La Actual Gestión Ambiental del municipio se evaluó en base a las respuestas de los directores de las divisiones, que comprenden diferentes áreas, que se describen en la tabla 5 .

Una vez definido el marco teórico y teniendo como base el Diagnostico se está en condición de proponer la Gestión ambiental del centro Poblado Mi Perú, basado en los programas propuestos y proyectos mencionado en la tabla 6

Para implementar correctamente la política ambiental, y además cumplir con los objetivos trazados, es necesario que el Municipio cuente con los recursos humanos y financieros adecuados, para un eficiente desempeño en cuanto al tema ambiental corresponde.

Cada programa ambiental definido previamente en la etapa de planificación, debe ser acompañado por un programa de seguimiento, el cual va permitir medir el cumplimiento de los objetivos trazados inicialmente.

Todos los años el municipio a través de su Unidad Ambiental revisará la Gestión Ambiental, considerando la necesidad de realizar cambios a la política de medio ambiente, a los objetivos y metas. Incluirá al menos el grado de cumplimiento de los objetivos y metas. Toda revisión será documentada, este documento contendrá las observaciones obtenidas, las conclusiones y recomendaciones realizadas luego de la evaluación, para la mejora del sistema. Dicha revisión consiste en realizar evaluaciones periódicas, comparando el comportamiento ambiental del sistema, con las políticas, objetivos y metas definidas en la planificación, con el fin de reconocer oportunidades de mejora.

\section{CONCLUSIONES}

- Según el diagnóstico de la actual gestión del municipio, existe poca o casi nula coordinación entre las áreas para tratar el tema ambiental, poca inclusión de la participación ciudadana, nula inclusión de los factores ambientales, profunda falta de planificación en aspectos ambientales, falta de documentación de los mismos, y además escasa presencia de programas ambientales.

Tabla 5. Diagnóstico ambiental del municipio

\begin{tabular}{|l|l|l|l|l|l|}
\hline \hline Tema & \multicolumn{1}{|c|}{ Punción } & \multicolumn{1}{|c|}{ Documentación } & \multicolumn{1}{c|}{$\begin{array}{c}\text { Objetivos y } \\
\text { Metas }\end{array}$} & \multicolumn{1}{c|}{$\begin{array}{c}\text { Medición y } \\
\text { Evaluación }\end{array}$} \\
\hline Participación Ciudadana & No existe & No existe & No definida & No existe & Según su importancia \\
\hline Denuncias Ambientales & No existe & Dispersa & No definida & No existe & Según su importancia \\
\hline Fiscalización Ambiental & Según su importancia & Dispersa & No definida & No existe & No existe \\
\hline Educación y Capacitación & No existe & No existe & No existe & No existe & No existe \\
\hline Coordinación Interna & No definida & No existe & No existe & No existe & No existe \\
\hline Coordinación Externa & No definida & Dispersa & No existe & No existe & Sogún su importancia existe \\
\hline Política Ambiental & No definida & No existe & No existe existe & & No existe \\
\hline Información a la Comunidad & No existe & & &
\end{tabular}

Tabla 6. Propuesta de Gestión Ambiental

\begin{tabular}{|c|c|c|c|c|}
\hline Programa & Objetivos & Metas & Legislación & Plan de Actividades \\
\hline $\begin{array}{l}\text { - Comité participativo de } \\
\text { Evaluación de Impacto } \\
\text { Ambiental } \\
\text { - Encuesta Percepción } \\
\text { Ciudadana } \\
\text { - Educación Ambiental }\end{array}$ & $\begin{array}{l}\text { Coordinación para el } \\
\text { análisis de los proyectos } \\
\text { que entra al EIA }\end{array}$ & $\begin{array}{l}\text { - Revisión de los } \\
\text { proyectos dentro del } \\
\text { plazo establecido } \\
\text { - Velar por el cuidado } \\
\text { del patrimonio } \\
\text { ambiental }\end{array}$ & $\begin{array}{l}\text { La Ley general del Ambiente- } \\
\text { Ley Na } 28611 \\
\text { La Ley Bases de la } \\
\text { descentralización - Ley Na } \\
27783\end{array}$ & $\begin{array}{l}\text { - Identificar de las áreas } \\
\text { Municipales que tienen mayor } \\
\text { injerencia en el tema ambiental } \\
\text { - Estructuración del comité. } \\
\text { - Elaboración de un calendario de } \\
\text { actividades } \\
\text { - Definición de responsabilidades } \\
\text { - Manejo de la información. } \\
\text { - Elaboración de boletines } \\
\text { informativos }\end{array}$ \\
\hline
\end{tabular}


- Solo 5 sectores de los 28 del centro poblado presentan factores ambientales favorables, se recomienda manejar responsablemente estos factores ambientales, puesto que el aumento de densidad urbana, influencia industrial o el cambio de uso de suelos, podría hacer bajar el nivel de calidad ambiental.

- El asentamiento humano Señor de Luren, que es el de mayor número de viviendas y habitantes de todo el centro poblado Mi Perú, muestra el índice de calidad más preocupante, y debe ser trabajado en el plan de actividades de la gestión ambiental municipal propuesta.

- Se sugiere a la municipalidad, aceptar y comprender la gran importancia que ha ido ganando con el tiempo la incorporación de variables ambientales, utilizando como principal herramienta la gestión ambiental municipal. Así como tomar en cuenta otros factores o variables ambientales para su inclusión en un futuro diagnóstico.

\section{REFERENCIAS BIBLIOGRÁFICAS}

Ágreda, E. (2000). El CEPMA SAN MARTÍN: Una experiencia de participación y concertación local para la gestión ambiental de Tarapoto. Lima: ITDG-Perú, 2000 x, 105 p.; ilus., mapas, planos. Recuperado de https://solucionespracticas.org.pe/ el-cepma-san-martin-una-experiencia-de-participacion-yconcertacion-local-para-la-gestion-ambiental-de-tarapoto

Alegre, A., \& Chirinos, L. (2001). Ordenanza de Gestión Ambiental. Tarapoto, San Martín. Recuperado de http:// www.mpsm.gob.pe/architrans/ORDENANZAS/2016/ ordenanza-municipal-023.pdf

ALEGRE, CH. M. (2002). Situación Actual y una Mirada al Futuro del Ambiente Urbano en el Perú. El Medio Ambiente en el Perú Año 2001. Instituto Cuánto. USAID. Ed. e Imp. Desa S.A. Edic. IEC para el Desarrollo. Lima Perú. 713 pp. http://www.cuanto.org/index.php?modulo=55

Bosque, J. (1992). La Enseñanza de los Sistemas de Información Geográfica. Actas del V Coloquio de Geografía Cuantitativa de la Universidad de Zaragoza, pp. 47-57.. Asociación de Geógrafos Españoles. Recuperado de http://tig.agegeografia.es//zaragoza92/1992_03_bosque.pdf

Cabrera, C., Maldonado, M., Arevalo, W., Pacheco, R., Giraldo, A., \& Loayza, S. (2012). Relaciones entre calidad ambiental y calidad de vida en Lima Metropolitana. Revista del Instituto de Investigación de la Facultad de Ingeniería Geológica, Minera, Metalúrgica y Geográfica, 47-52. Recuperado de https://revistasinvestigacion.unmsm.edu.pe/ index.php/iigeo/article/view/664
Carmona, A., \& Monsalve, J. (1999). Sistemas de Información Geográficos. Congreso de Ingeniería de Sistemas en la Universidad San Buenaventura de Medellín Colombia, pp. 44. Recuperado de https://dds.cepal.org/infancia/guiapara-estimar-la-pobreza-infantil/bibliografia/capitulo-IV/ Carmona Alvaro y Monsalve Jhon (1999) Sistemas de informacion geografica.pdf

González, E. (2001). Gestión Ambiental en Pequeños Municipios. En Revista Foro, 42 - Octubre, 2001. Pág. 57. Bogotá, Colombia.

Latorre Estrada, E. (2000). Herramientas para la Participación en Gestión Ambiental. Bogota: Editorial Prisma Asociados, 2000. $313 \mathrm{p}$.

López Vásquez, J. (2012). Problemática y propuesta de gestión ambiental en la ciudad de Laredo, Trujillo, Perú. Ciencia y Tecnología, 191-207. Obtenido de http://revistas.unitru.edu. pe/index.php/PGM/article/download/155/158

Ministerio del Ambiente. (Octubre de 2005). Ley N. ${ }^{\circ} 28611$ - Ley General del Ambiente. Lima, Perú. Recuperado de http:// www.minam.gob.pe/wp-content/uploads/2013/06/leygeneral-del-ambiente.pdf

Muncipalidad de San Isidro. (2008). Ordenanza de Gestión Ambiental. Lima. Recuperado de https://sinia.minam.gob. pe/download/file/fid/65047

Municipalidad de Mi Perú (2016). Municipalidad del Centro Poblado Nuestra Señora de las Mercedes de Mi Perú. Recuperado de http://munimiperu.gob.pe/

Muriel, R. (2006). Gestión Ambiental. Ide@sostenible: espacio de reflexión y comunicación en desarrollo sostenible, Año 3 No. 13, pp. 1-8. Recuperado de https:// upcommons.upc.edu/bitstream/handle/2099/1110/13 GestAmbientalRafaelMuriel_cast.pdf

Sarde, P. (1999). ISO 14000 en la Gestión Ambiental. Documento mimeografiado de la Especialización en Gestión Ambiental de la Universidad Nacional de Colombia. Medellín. 1999 p. 3.

Senamhi. (2018). Servicio Nacional de Meteorología e Hidrología del Perú. Recuperado de https://www.senamhi.gob.pe/

Serrano, J. L. (1997). Principios filosóficos de la gestión ambiental, en J. Ballesteros y J. Pérez Adán (eds.): Sociedad y medio ambiente, Trotta, Madrid, 1997, pp. 323-338. Recuperado de https://dialnet.unirioja.es/servlet/ libro? codigo $=503306$

Spiegel, J. \& Yassi, A. Journal of Medical Systems (1997) 21: 275. https://doi.org/10.1023/A:1022868507945

Toledo, B. (2018). La importancia de la gestión ambiental municipal. Estudio de caso: municipios del departamento de Santa Ana, El Salvador. INVENTUM, 12(23), 22-34. https:// doi.org/10.26620/uniminuto.inventum.12.23.2017.22-34 